\title{
Understanding the phylogeographic patterns of European hedgehogs, Erinaceus concolor and $E$. europaeus using the $\mathrm{MHC}$
}

\author{
KT Berggren ${ }^{1}$, H Ellegren ${ }^{1}$, GM Hewitt ${ }^{2}$ and JM Seddon ${ }^{1,3}$ \\ ${ }^{1}$ Department of Evolutionary Biology, EBC, Uppsala University, Uppsala, Sweden; ${ }^{2}$ School of Biological Sciences, University of East \\ Anglia, Norwich, UK; ${ }^{3}$ School of Veterinary Science, The University of Queensland, St Lucia, QLD, Australia
}

\begin{abstract}
The genome of the European hedgehog, Erinaceus concolor and E. europaeus, shows a strong signal of cycles of restriction to glacial refugia and postglacial expansion. Patterns of expansion, however, differ for mitochondrial DNA (mtDNA) and preliminary analysis of nuclear markers. In this study, we determine phylogeographic patterns in the hedgehog using two loci of the major histocompatibility complex $(\mathrm{MHC})$, isolated for the first time in hedgehogs. These genes show long persistence times and high polymorphism in many species because of the actions of balancing selection. Among 84 individuals screened for variation, only two $D Q A$ alleles were identified in each
\end{abstract}

species, but $10 D Q B$ alleles were found in $E$. concolor and six in $E$. europaeus. A strong effect of demography on patterns of $D Q B$ variability is observed, with only weak evidence of balancing selection. While data from mtDNA clearly subdivide both species into monophyletic subgroups, the $\mathrm{MHC}$ data delineate only $E$. concolor into distinct subgroups, supporting the preliminary findings of other nuclear markers. Together with differences in variability, this suggests that the refugia history and/or expansion patterns of $E$. concolor and $E$. europaeus differ.

Heredity (2005) 95, 84-90. doi:10.1038/sj.hdy.6800694

Published online 20 April 2005

Keywords: balancing selection; $D Q A ; D Q B$; hedgehog; $\mathrm{MHC}$; postglacial colonisation

\section{Introduction}

It has been shown that the genomes of many European species of plants and animals have been affected by the dramatic climate changes associated with cyclical glacial and interglacial periods (Hewitt, 1996, 1999; Comes and Kadereit, 1998; Taberlet et al, 1998). During colder periods, temperate species have gone extinct, dispersed to new locations or survived in refugia, expanding out in postglacial periods (Hewitt, 2000). Mitochondrial DNA (mtDNA) studies show that the genomes of European hedgehogs bear the marks of glacially induced patterns of repeated expansion and contraction (Santucci et al, 1998; Seddon et al, 2001). It is likely that the two parapatric species of European hedgehogs, the whitebreasted Erinaceus concolor in eastern Europe and the brown-breasted E. europaeus in western Europe (Reeve, 1994), found refuge in Iberia, Italy and the Balkans, expanding northwards in interglacial periods (Santucci et al, 1998; Seddon et al, 2001). The deep split between E. concolor and E. europaeus and the further subdivisions within the species (Santucci et al, 1998; Seddon et al, 2001) show that there have been strong effects of climate on the distribution of genetic variation of the hedgehogs. However, nuclear intron sequence data show a different

Correspondence: J Seddon, School of Veterinary Science, University of Queensland, St Lucia, QLD 4072, Australia.

E-mail: j.seddon1@uq.edu.au

Received 22 January 2004; accepted 18 March 2005; published online 20 April 2005 picture of divergence between postulated refugial groups in E. concolor but not E. europaeus. The addition of further loci is required to determine if this pattern is consistent across nuclear markers.

The class II major histocompatibility complex (MHC) genes include loci that display unusually high levels of polymorphism (reviewed in, eg, Potts and Wakeland, 1990; Meyer and Thomson, 2001). This high level of variation is consequent on the MHC molecules intrinsic role in the normal functioning of the immune system, with polymorphism concentrated in the peptide binding region (PBR) sites involved in pathogen recognition (Hughes and Nei, 1988, 1989). Polymorphism is thought to be maintained by balancing selection primarily driven by pathogen-based selection, through heterozygote advantage (Doherty and Zinkernagel, 1975; Hughes and Nei, 1988) or frequency-dependent selection (Bodmer, 1972; Takahata and Nei, 1990). The action of balancing selection also results in the long persistence times of MHC alleles. Transspecies polymorphism, in which alleles show relatedness extending beyond speciation events, indicates balancing selection in and from the distant past (Klein, 1987; Figueroa et al, 1988; Garrigan and Hedrick, 2003). Hence, MHC genes are likely to provide a highly polymorphic system of alleles, many of which will predate the glacially induced bottlenecks of the European hedgehog. The aim of this study, therefore, is to assess the level of MHC variation in European hedgehogs to determine if the pattern of variation provides consistent differences in nuclear loci from the mtDNA patterns, furthering our understanding of the history of these species. 


\section{Materials and methods}

\section{Samples}

A total of 84 samples from European hedgehogs, 22 E. concolor and 62 E. europaeus, were screened for variation in this study. The samples represent a subset of those used in previous mitochondrial studies (Seddon et al, 2001), and they were selected to represent high mtDNA divergence and widespread locations. The locations of the samples by country are indicated in Table $1 a$ and $b$, with more specific information available in online supplementary information.

\section{Laboratory procedures}

Genomic DNA was extracted by standard phenol/ chloroform procedures (Sambrook et al, 1989). For $D Q A$, PCR amplification employed a published forward primer (Slade et al, 1993) and a new reverse primer within the intron close to exon 2 (5'-CTCTGTCACTG AAGAAGTATGGA-3'). For $D Q B$, two primers were designed within exon 2 in regions conserved across species: 5'-GATTTCGTGTACCAGTTYAAGG-3' and 5'TTGTRTCTGCASACCGTGTC-3'. A lack of conserved regions in DRB exon 2 precluded its use in this study.

The PCR reactions contained $0.2 \mu \mathrm{M}$ of each primer, $0.1 \mathrm{mM}$ dNTPs, $2.0 \mathrm{mM} \mathrm{MgCl} 2,0.05 \mathrm{U} / \mu \mathrm{l}$ AmpliTaq Gold (Applied Biosystems) Taq polymerase in $1 \times$ AmpliTaq buffer and approximately $1 \mathrm{ng} \mathrm{DNA} / \mu \mathrm{l}$ reaction mix. The PCR programme had an initial denaturation of $95^{\circ} \mathrm{C}$ for $7 \mathrm{~min}, 35$ cycles of $95^{\circ} \mathrm{C}$ for $40 \mathrm{~s}, 50^{\circ} \mathrm{C}$ for $1 \mathrm{~min}$ and $72{ }^{\circ} \mathrm{C}$ for $1 \mathrm{~min}$ and $30 \mathrm{~s}$, and a final extension of $72^{\circ} \mathrm{C}$ for $10 \mathrm{~min}$.

All samples were screened for polymorphism at exon 2 of $D Q A$ and $D Q B$ using single-strand conformation polymorphism (SSCP) (Orita et al, 1989). Standard 12\% polyacrylamide gels were run at $4^{\circ} \mathrm{C}(D Q A$ and $D Q B)$ or $12^{\circ} \mathrm{C}(D Q B)$, with $2 \%$ glycerol added for $D Q A$. For $D Q B$, a subset of homozygous samples, representing each configuration pattern on the SSCP gels, as well as all heterozygous samples were selected for sequencing. For the DQA locus, which appeared monomorphic on SSCP, all samples were sequenced after more than one allele was revealed by preliminary sequencing. PCR products were purified by EXOsap-IT (USB), and sequenced using a MegaBACE 1000 instrument (Amersham Biosciences).

Heterozygous samples in which one or both $D Q B$ alleles were absent in homozygous form were cloned, unless only one site differed between the two alleles. PCR products were purified using the QIAquick protocol (Qiagen), ligated using the pGEM-T Vector System (Promega) and transformed with JM109 Escherichia colicompetent cells (Promega). For each sample, a total of 10-12 colonies were sequenced. Alleles seen only in heterozygous form were confirmed when at least three cloned sequences showed concordance or, in one case, with two cloned sequences of each allele, differing only at sites previously identified as heterozygous. Sequences have been deposited in GenBank (Accession numbers: AY929164-AY929183).

\section{Data analyses}

$D Q A$ and $D Q B$ hedgehog sequences were aligned with related sequences in other mammalian species. For $D Q A$, the hedgehog sequences formed a monophyletic clade in a neighbour-joining tree (Jukes-Cantor distances, 1000 bootstrap iterations), more closely related to $D Q A$ sequences of other mammals than to other MHC alpha genes. A similar pattern was seen with hedgehog $D Q B$ sequences most closely related to mammalian $D Q B$ sequences, confirming that the expected genes have been amplified.

After translation, the absence of stop codons within the amino-acid sequences of $D Q A$ and $D Q B$ suggested that the sequences encoded functional proteins. No more than two alleles were found in any individual, indicating that single-copy loci had been amplified. PBR sites were identified for $D Q A$ and $D Q B$ according to the predictions in Brown et al $(1988,1993)$, who modelled the PBR sites in human class II MHC molecules.

The number of nonsynonymous substitutions per nonsynonymous site $\left(d_{N}\right)$ and the number of synonymous substitutions per synonymous site $\left(d_{\mathrm{S}}\right)$ were calculated in MEGA v 2.1 (Kumar et al, 2001) using the Nei-Gojobori method with a Jukes-Cantor correction and the standard error estimated by bootstrapping (500 iterations). The mean pairwise number of nucleotide differences was calculated in MEGA v 2.1 (Kumar et al, 2001) and the nucleotide diversity was calculated in DnaSP v 4.0 (Rozas et al, 2003). Neighbour-joining trees were constructed for all hedgehog $D Q A$ and $D Q B$ alleles using Jukes-Cantor distance measures with 1000 bootstrap replicates in MEGA v 2.1 (Kumar et al, 2001).

The number of MHC alleles was compared with the number of mitochondrial types (Seddon et al, 2001). To control for biases in the level of variation due to the selection of samples, 10000 data sets of 22 E. concolor and 62 E. europaeus individuals were created by resampling with replacement. The $\mathrm{MHC} / \mathrm{mtDNA}$ ratio was calculated for each data set and the mean values compared using two-tailed $t$-tests.

\section{Results}

\section{$D Q A$ sequences}

Low levels of diversity were found at $D Q A$ in the hedgehog. In E. concolor, only two alleles were identified: ErcoDQ $A^{*} 01$, which was found in all samples north of the Bosphorus, and ErcoDQA*02, which was identified in all samples found south of the Bosphorus (Table 1a). One nonsynonymous substitution, located within a PBR site, distinguishes the two alleles. In E. europaeus two alleles were found. EreuDQA*01, which was identical to ErcoDQ $A^{*} 01$, was identified in many samples across a wide area, while EreuDQ $A^{*} 02$ was found only twice in homozygous form and once in heterozygous form among individuals from Estonia (Table 1b). One nonsynonymous substitution, located within a non-PBR site, distinguishes the two alleles. The phylogenetic relationship among the alleles is shown in online supplementary information.

\section{$D Q B$ sequences}

The $D Q B$ locus displayed higher diversity than $D Q A$ in the European hedgehogs. In E. concolor, 10 alleles were found, identified as ErcoDQB*01 to ErcoDQB*10 (Table 1a). These alleles are distinguished by 14 substitutions at a total of 13 polymorphic sites with eight amino-acid changes at seven polymorphic sites. 
Table 1 Distribution of $D Q A$ and $D Q B$ alleles by locality for (a) E. concolor and (b) E. europaeus

\begin{tabular}{|c|c|c|c|c|c|c|}
\hline \multirow[t]{2}{*}{ Country } & \multirow[t]{2}{*}{$\mathrm{N}$} & \multicolumn{2}{|c|}{ DQA } & \multicolumn{2}{|l|}{ DQB } & \multirow[t]{2}{*}{$m t D N A$} \\
\hline & & $\mathrm{H}_{o b s}$ & Alleles & $\mathrm{H}_{o b s}$ & Alleles & \\
\hline \multicolumn{7}{|l|}{ (a) E. concolor } \\
\hline Estonia & 1 & - & $* 01$ & - & $* 01$ & $\mathrm{C} 1$ \\
\hline Poland & 1 & - & $* 01$ & 1.0 & $* 01, * 05$ & $\mathrm{C} 1$ \\
\hline Austria/Hungary & 5 & - & *01 & 0.60 & ${ }^{*} 01,{ }^{*} 05, * 06, * 07$ & $\mathrm{C} 1$ \\
\hline Italy & 4 & - & $* 01$ & 0.50 & ${ }^{*} 01, * 04, * 06, * 08$ & $\mathrm{C} 1$ \\
\hline Croatia/Serbia & 2 & - & ${ }^{*} 01$ & 0.50 & ${ }^{*} 01, * 03, * 04$ & $\mathrm{C} 1$ \\
\hline Greece/Turkey (Thrace) & 4 & - & ${ }^{*} 01$ & 0.50 & $*^{*} 03, * 06, * 09$ & $\mathrm{C} 1$ \\
\hline Israel/Turkey (Anatolia) & 5 & - & ${ }^{*} 02$ & 0.20 & ${ }^{*} 02, * 10$ & $\mathrm{C} 2$ \\
\hline \multicolumn{7}{|l|}{ (b) E. europaeus } \\
\hline UK & 6 & - & $*^{*} 01$ & - & ${ }^{*} 01$ & E2 \\
\hline Denmark/Norway/Sweden & 5 & - & ${ }^{*} 01$ & 0.40 & ${ }^{*} 01,{ }^{*} 02$ & E1 \\
\hline Estonia/Russia & 7 & 0.14 & $* 01, * 03$ & 0 & $* 01, * 02$ & E1 \\
\hline Netherlands & 7 & - & $* 01$ & 0.43 & $* 01, * 03$ & E1, E2 \\
\hline Germany & 11 & - & $* 01$ & 0.09 & $* 01, * 04$ & E1 \\
\hline Switzerland & 6 & - & $* 01$ & 0.50 & $* 01,{ }^{*} 03$ & E1, E2 \\
\hline France & 6 & - & $* 01$ & 0 & $* 01, * 03$ & E2 \\
\hline Spain/Portugal & 6 & - & $* 01$ & 0.17 & $* 01, * 05$ & E2 \\
\hline Italy/Corsica & 8 & - & $* 01$ & 0 & ${ }^{*} 01,{ }^{*} 02,{ }^{*} 06$ & E1, E3 \\
\hline
\end{tabular}

$N=$ number of samples; $H_{\mathrm{obs}}=$ observed proportion of heterozygotes. mtDNA haplotype groups are given according to Seddon et al (2001).

Although rare alleles were found across most of the distribution area of E. concolor, the highest number of alleles was found in central Europe, in the region of the Alps (Figure 1), although increased sampling through the postulated refugial region of the Balkans, where greater variation may exist, might change this. As for $D Q A$, there is no overlap in allele distribution across the Bosphorous. In E. europaeus, six alleles were identified (EreuDQB*01-EreuDQB*06; Table 1b), defined by nine substitutions at nine polymorphic sites and six aminoacid changes at six polymorphic sites. As for $D Q A$, one allele is shared between the two species (ErcoDQB*01 and EreuDQB*01). Allele EreuDQ $B^{*} 01$ seems fixed in many regions, and importantly, allele Ereu $D Q B^{*} 03$ is found across the boundary of two mitochondrial clades (Figure 1).

At the $D Q B$ locus, E. concolor shows greater variation than E. europaeus in the number of alleles and nucleotide diversity, which is significantly higher in $E$. concolor $(E$. concolor $0.025 \pm 0.0013, E$. europaeus $0.0077 \pm 0.0012$, $t=9.8, P<0.005)$. Higher heterozygosity levels are also noted in E. concolor (E. concolor $45.5 \%$, E. europaeus $16.1 \%$ ). However, the lower values in E. europaeus are probably strongly influenced by allele fixation in north and far western Europe. Although more alleles are found in E. concolor, the alleles are not more divergent, as shown by the lack of a significant difference between the two species in mean pairwise number of nucleotide differences ( $E$. concolor $5.62 \pm 1.51$, E. europaeus $4.20 \pm 1.38$, $t=0.69, P>0.05)$.

\section{Indicators of evolution at $D Q B$}

The presence of alleles shared between the species suggests the long-term action of balancing selection and this is further supported by the absence of speciesspecific clades in a phylogenetic analysis of $D Q B$ alleles (Figure 2). In contrast, there is little support for balancing selection in the patterns of nonsynonymous substitutions. In the presence of balancing selection, values $d_{\mathrm{N}}$ and $d_{\mathrm{S}}$ for PBR and non-PBR sites (Table 2) are expected to show a $d_{\mathrm{N}} / d_{\mathrm{S}}$ ratio greater than one at the functionally important PBR sites and a $d_{\mathrm{N}} / d_{\mathrm{S}}$ ratio less than one at non-PBR sites. This pattern is noted for E. europaeus but not for E. concolor. However, the low number of variable PBR sites results in high standard errors when calculating $d_{\mathrm{N}}$ and $d_{\mathrm{S}}$ values for PBR sites.

For both species, there is a strong bias towards PBR variability in the $5^{\prime}$ region of the exon. All variable PBR sites in E. concolor and six out of seven variable PBR sites in E. europaeus occur in the $5^{\prime}$ sequence region that encodes the $\beta$-floor of the peptide binding cleft, with no or very low variability at PBR sites in the $\alpha$-helix. Furthermore, there is higher nucleotide divergence calculated over all sites in the $\beta$ than the $\alpha$ regions, reaching significance in E. europaeus (Jukes-Cantor distance: $E$. concolor $-\beta 0.041 \pm 0.013$ and $\alpha 0.014 \pm$ $0.010, t=1.6, P>0.05$; E. europaeus $-\beta 0.033 \pm 0.012$ and $\alpha 0.0051 \pm 0.0051, t=2.1, P<0.05)$.

\section{Number of $D Q B$ alleles compared with mtDNA results}

To obtain a measure of the relative level of variation at the $D Q B$ locus, the number of alleles was compared to the number of mtDNA types (Seddon et al, 2001) (Table 3). It is clear that the level of MHC variation in comparison to the level of mtDNA variation is lower in E. europaeus than in E. concolor. While this could be biased by the selection of samples for high mtDNA variation, resampling with replacement shows that the $\mathrm{MHC} /$ mtDNA ratio remains significantly lower in E. europaeus $(t=583, P<0.0001)$. The level of mtDNA variation is similar between the two species and so the observed $\mathrm{MHC} / \mathrm{mtDNA}$ difference is likely to reflect lower MHC variation in E. europaeus.

\section{Discussion}

\section{Demographic effects on $\mathrm{MHC}$ variation}

The overall distribution of MHC alleles in hedgehogs across Europe is consistent with patterns observed for mitochondrial markers and nuclear introns, suggesting 


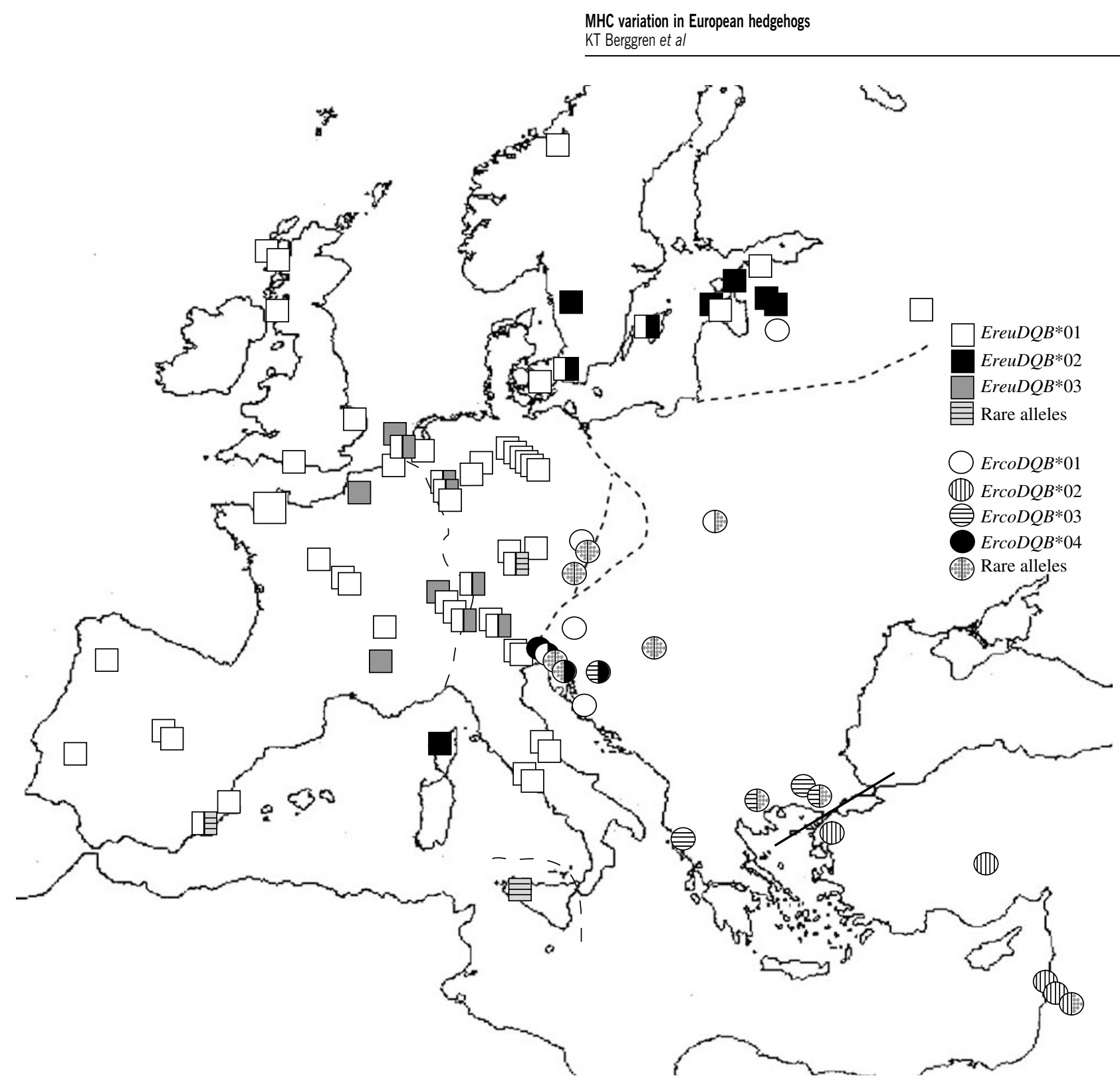

Figure 1 Distribution of $D Q B$ alleles among European hedgehogs, E. concolor and E. europaeus. Each figure (square, E. europeaus; circle, E. concolor) represents the genotype of one sample. Heterozygous samples are divided into two. Alleles with an intraspecific frequency of less than $10 \%$ are grouped as 'rare alleles'. The approximate separation of mitochondrial clades (Seddon et al, 2001) is shown by dashed lines in E. europaeus and by an unbroken line shows in E. concolor. The dotted lines indicate the boundaries between the two species.

that demography and range changes associated with cycles of restriction to glacial refugia and postglacial expansion have been the predominant force in shaping the patterns of MHC variation.

There are obvious similarities between the results from this study and previous analyses of mtDNA (Seddon et al, 2001), in terms of the geographic distribution of alleles. In particular, a deep subdivision in E. concolor is seen for both markers, coinciding with the Bosphorus. As for the mtDNA, there are no shared MHC alleles across this barrier, suggesting that there has been a lack of gene flow and effective isolation of these regions for a considerable period of time. One may broadly discern a similar western, central, eastern and Balkan genome pattern across Europe in the $D Q B$ allele data (Figure 1) as in the mtDNA (Seddon et al, 2001), but it is less distinct than the mtDNA. Other similarities are seen, with EreuDQB*02 found in Corsica as well as Scandinavia and Estonia, mimicking the long-distance colonisation in hedgehogs seen with mtDNA, and, in a manner similar to the divergent mtDNA haplotype identified only in Sicily, a unique allele (EreuDQB*06) was restricted to Sicily in this study (Seddon et al, 2001). Despite these apparent similarities, there are two important differences between the patterns of MHC and mtDNA variation.

Firstly, while the amount of mtDNA variation is similar between the two species (Seddon et al, 2001), there is more MHC variation in E. concolor than in $E$. europaeus. There are a total of $10 \mathrm{DQB}$ alleles found among the 22 samples of E. concolor, while there are only six alleles identified among the 62 samples of E. europaeus. A contingency $\chi^{2}$ test shows these ratios to be significantly different $\left(\chi^{2}=8.15, P<0.01\right)$, despite the relatively small sample sizes. Furthermore, the relative level of MHC variation at the $D Q B$ locus in comparison to the mtDNA variation is more than three times higher 
in E. concolor. This finding supports the previous analysis of two nuclear introns in 23 hedgehog samples, in which higher nuclear diversity was noted for E. concolor. This suggests that the differences in variation between the species relate to the nuclear genome overall and not specifically to the MHC.

Secondly, postulated refugial clades within E. europaeus are not concordant among nuclear and mtDNA markers. Data from mtDNA (Seddon et al, 2001) clearly subdivide both species into monophyletic subgroups, suggesting separate past refugia and postglacial coloni-

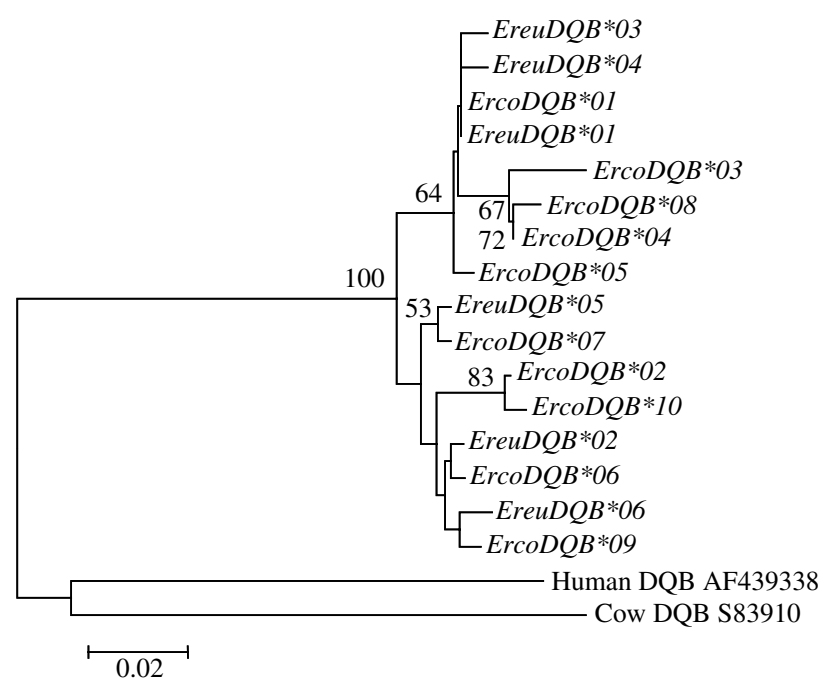

Figure 2 Neighbour-joining tree of the $15 D Q B$ alleles identified in E. concolor and E. europaeus. The tree is based on Jukes-Cantor distances. Bootstrap values from 1000 iterations are shown where values exceed $50 \%$. $D Q B$ sequences from human and cow are included as outgroups. sation routes. However, the nuclear intron results from the same study and the results from this MHC study, for both $D Q A$ and $D Q B$, although limited by sample size, delineates only $E$. concolor into distinct subgroups.

Given there is little evidence for sex-biased dispersal in hedgehogs (Reeve, 1994), one possible explanation for the inconsistent pattern in mtDNA and nuclear loci is that the sorting of alleles is a result of repeated refugial separation. The similar level of intraspecific mtDNA divergence within E. concolor and E. europaeus suggests that the more recent refugial separations occurred at the same time in both species, with recent bottlenecks associated with contractions to the refugia accounting for the subdivision of mtDNA. However, the results from this MHC study and the previous nuclear intron study (Seddon et al, 2001) suggest additional older separation in refugia in E. concolor, allowing sorting of nuclear alleles, the signal of which has remained in the nuclear genome of E. concolor, while being eroded by more recent cycles in E. europaeus. The suggestion proposed by Seddon et al (2001) of a more severe bottleneck in the founding of E. concolor compared to E. europaeus is rendered unlikely by the relatively high level of MHC variation in E. concolor, which suggests that a large population size has been maintained during glacial periods.

A second explanation is a difference in past expansion patterns between the species. A more rapid expansion from refugia for E. europaeus would have resulted in a stronger founder effect and thus more severe loss of genetic variation (Hewitt, 1996; Ibrahim et al, 1996). If such rapid expansion occurred repeatedly over several glacial cycles, then a reduction in both MHC and mtDNA may be expected. In contrast, slower expansion patterns for E. concolor in the past could have maintained MHC variation. An extreme loss of genetic variation, by refugial bottlenecks or founder events, will obliterate

Table 2 Synonymous $\left(d_{\mathrm{S}}\right)$ and nonsynonymous $\left(d_{\mathrm{N}}\right)$ changes at peptide binding region and nonpeptide binding region sites in hedgehog exon $2 D Q B$ alleles

\begin{tabular}{|c|c|c|c|c|c|c|c|c|c|}
\hline \multirow[t]{2}{*}{ Species } & \multirow[t]{2}{*}{$\mathrm{N}$} & \multicolumn{4}{|c|}{ Peptide binding region } & \multicolumn{4}{|c|}{ Nonpeptide binding region } \\
\hline & & $\mathrm{d}_{N}$ & $\mathrm{~d}_{S}$ & & & $\mathrm{~d}_{N}$ & $\mathrm{~d}_{S}$ & & \\
\hline E. concolor & 10 & $0.037 \pm 0.025$ & $0.050 \pm 0.053$ & 0.75 & ND & $0.027 \pm 0.013$ & $0.029 \pm 0.018$ & 0.92 & ND \\
\hline E. europaeus & 6 & $0.040 \pm 0.023$ & $0.000 \pm 0.000$ & ND & * & $0.019 \pm 0.012$ & $0.027 \pm 0.021$ & 0.71 & ND \\
\hline
\end{tabular}

$n=$ number of alleles; $\mathrm{ND}=$ not determined.

$d_{\mathrm{S}}$ and $d_{\mathrm{N}}$ values were calculated using the Nei-Gojobori method with a Jukes-Cantor correction. Standard errors were estimated by 500 bootstrap iterations. In all, 14 PBR codons and 48 non-PBR codons were analysed.

Statistical significance assessed by one-tailed $t$-test: ${ }^{*}, P<0.05$.

Table 3 Comparison between $\mathrm{MHC}(D Q B)$ and mitochondrial variation in hedgehogs

\begin{tabular}{lrcccc}
\hline Species by mtDNA group & $\mathrm{N}$ & No. of DQB alleles & No. of $m$ tDNA types & MHC/mtDNA & Unbiased MHC/mtDNA \\
\hline E. concolor & 22 & 10 & 22 & 0.455 & $0.608 \pm 0.0008$ \\
C1 & 17 & 8 & 17 & 0.471 & \\
C2 & 5 & 2 & 5 & 0.127 & $0.138 \pm 0.0002$ \\
& & 7 & 55 & 0.105 & \\
E. europaeus & 62 & 4 & 38 & 0.176 \\
E1 & 39 & 3 & 17 & \\
E2 & 23 & &
\end{tabular}

Unbiased MHC/mtDNA ratios were estimated by creating 10000 data sets of 22 E. concolor and 62 E. europaeus individuals by resampling with replacement. MtDNA groupings (E1, E2, C1, C2) are based on Seddon et al (2001). 
the signal of previous events. Hence, the loss of variability at mtDNA but not at nuclear DNA indicates that the recent bottleneck(s) were mild, affecting mtDNA, a marker sensitive to the effects of population size reductions because it has an effective population size one-quarter that of nuclear DNA, but not resulting in obvious reduction of nuclear variability.

\section{MHC variation and effects of balancing selection}

Although transspecies polymorphism in the hedgehog MHC suggests the action of balancing selection, a conflicting signal is given at the nucleotide level by an unexpectedly low $d_{\mathrm{N}} / d_{\mathrm{S}}$ ratio in E. concolor. There are several explanations to explain an apparent reduction in selection. Firstly, there is a possibility that PBR sites may differ between different species and not reflect the predicted models of humans on which these calculations are based. This does not seem likely considering that the overall structure is often very conserved between species (Trowsdale, 1995). Secondly, environmental and behavioural factors that affect the strength of balancing selection have in some cases been proposed to account for low levels of MHC polymorphism (Slade, 1992; Murray et al, 1995; Ellegren et al, 1996). In hedgehogs, hibernation, in which body temperature and many physiological systems, including immune function, are greatly suppressed (Boyer and Barnes, 1999; Burton and Reichman, 1999; Nedergaard and Cannon, 1990) and may affect the strength of balancing selection. Thirdly, the inconsistent pattern between the $\beta$-floor and the $\alpha$ helix encoding regions may indicate site-specific selection pressure as seen in the estuarine fish (Fundulus heteroclitus; Cohen, 2002). However, a higher $d_{S}$ value in the $\beta$-floor region than for the $\alpha$-helix region suggests different evolutionary histories. Similar patterns have been noted in DRB sequences (Gyllensten et al, 1991; Schwaiger et $a l, 1993$ ) and a conserved recombination motif, present also in the hedgehog $D Q B$ sequences, has been identified (Wu et al, 1986).

\section{Conclusions}

The use of nuclear markers in addition to mtDNA has allowed us to draw more detailed conclusions about the demographic changes associated with the glacial refugia and postglacial expansion events. mtDNA is widely used in phylogeographic studies (reviewed by Taberlet et al, 1998; Hewitt, 1999), yet this marker is particularly sensitive to reductions in the effective population size and only provides information about the maternal line. In the European hedgehog, the use of mtDNA alone suggested distinct lineages in E. europaeus that were not supported by nuclear data. However, the clear separation of E. concolor into two clades, north and south of the Bosphorous, is noted with both MHC and mtDNA. Hence, these two species have different refugial histories, with inferred differences in population size or expansion patterns. This additional demographic information gained demonstrates that the use of more than one marker type is to be encouraged in phylogeographic studies.

\section{Acknowledgements}

We would like to thank Fiammetta Santucci, Nigel Reeve and the many people who have provided hedgehog samples for earlier research at UEA Norwich funded by the Leverhulme Trust. HE is a Royal Academy of Sciences Research Fellow supported by a grant from Knut and Alice Wallenberg foundation.

\section{References}

Bodmer WF (1972). Evolutionary significance of the HL-A system. Nature 237: 139-145.

Boyer BB, Barnes BM (1999). Molecular and metabolic aspects of mammalian hibernation. Bioscience 49: 713-724.

Brown JH, Jardetzky T, Gorga JC, Stern LJ, Urban RG, Strominger JL et al (1993). Three-dimensional structure of the human class II histocompatibility antigen HLA-DR1. Nature 364: 33-39.

Brown JH, Jardetzky T, Saper MA, Samraoui B, Bjorkman PJ, Wiley DC (1988). A hypothetical model of the foreign antigen binding site of class II histocompatibility molecules. Nature 332: 845-850.

Burton RS, Reichman OJ (1999). Does immune challenge affect torpor duration? Funct Ecol 13: 232-237.

Cohen S (2002). Strong positive selection and habitat-specific amino acid substitution patterns in MHC from an Estuarine fish under intense pollution stress. Mol Biol Evol 19: 1870-1880.

Comes HP, Kadereit JW (1998). The effect of Quarternary climatic changes on plant distribution and evolution. Trends Plant Sci 3: 432-438.

Doherty PC, Zinkernagel RM (1975). Enhanced immunological surveillance in mice heterozygous at the $\mathrm{H}-2$ gene complex. Nature 256: 50-52.

Ellegren H, Mikko S, Wallin K, Andersson L (1996). Limited polymorphism at major histocompatibility complex (MHC) loci in the Swedish moose A. alces. Mol Ecol 5: 3-9.

Figueroa F, Gunther E, Klein J (1988). MHC polymorphism predating speciation. Nature 335: 265-267.

Garrigan D, Hedrick PW (2003). Detecting adaptive molecular polymorphism: lessons from the MHC. Evolution 57: 1707-1722.

Gyllensten UB, Sundvall M, Erlich HA (1991). Allelic diversity is generated by intraexon sequence exchange at the DRB1 locus of primates. Proc Natl Acad Sci USA 88: 3686-3690.

Hewitt GM (1996). Some genetic consequences of ice ages, and their role in divergence and speciation. Biol J Linn Soc 58 247-276.

Hewitt GM (1999). Post-glacial re-colonization of European biota. Biol J Linn Soc 68: 87-112.

Hewitt GM (2000). The genetic legacy of the Quaternary ice ages. Nature 405: 907-913.

Hughes L, Nei M (1988). Pattern of nucleotide substitution at major histocompatibility complex class I loci reveals overdominant selection. Nature 335: 167-170.

Hughes L, Nei M (1989). Nucleotide substitution at major histocompatibility complex class II loci: evidence for overdominant selection. Proc Natl Acad Sci USA 86: 958-962.

Ibrahim KM, Nichols RA, Hewitt GM (1996). Spatial patterns of genetic variation generated by different forms of dispersal during range expansion. Heredity 77: 282-291.

Klein J (1987). Origin of major histocompatibility complex polymorphism: the trans-species hypothesis. Hum Immunol 19: $155-162$.

Kumar S, Tamura K, Jakobsen IB, Nei M (2001). MEGA2: Molecular Evolutionary Genetics Analysis software. Bioinformatics 17: 1244-1245.

Meyer D, Thomson G (2001). How selection shapes variation of the human major histocompatibility complex: a review. Ann Hum Genet 65: 1-26.

Murray BW, Malik S, White BN (1995). Sequence variation at the major histocompatibility complex locus $D Q \beta$ in Beluga Whales (Delphinapterus leucas). Mol Biol Evol 12: 582-593. 
Nedergaard J, Cannon B (1990). Mammalian hibernation. Philos Trans $R$ Soc Lond Ser B 326: 669-686.

Orita M, Suzuki Y, Sekiya T, Hayashi K (1989). Rapid and sensitive detection of point mutations and DNA polymorphisms using the polymerase chain reaction. Genomics 5: 874-879.

Potts WK, Wakeland EK (1990). Evolution of diversity at the major histocompatibility complex. Trends Ecol Evol 5: 181-187.

Reeve N (1994). Hedgehogs. T \& AD Poyser (Natural History): London.

Rozas J, Sánchez-DelBarrio JC, Messeguer X, Rozas R (2003). DnaSP, DNA polymorphism analyses by the coalescent and other methods. Bioinformatics 19: 2496-2497.

Sambrook J, Fritsch EF, Maniatis T (1989). Molecular Cloning a Laboratory Manual, 2nd edn. Cold Spring Harbor Laboratory Press: New York.

Santucci F, Emerson BC, Hewitt GM (1998). Mitochondrial DNA phylogeography of European hedgehogs. Mol Ecol 7: 1163-1172.

Schwaiger F-W, Weyers E, Epplen C, Brün J, Ruff G, Crawford A, Epplen JT (1993). The paradox of MHC-DRB exon/intron evolution: $\alpha$-helix and $\beta$-sheet encoding regions diverge while hypervariable intronic simple repeats coevolve with $\beta$-sheet codons. J Mol Evol 37: 260-272.
Seddon JM, Santucci F, Reeve NJ, Hewitt GM (2001). DNA footprints of European hedgehogs, Erinaceus europaeus and E. concolor: Pleistocene refugia, postglacial expansion and colonization routes. Mol Ecol 10: 2187-2198.

Slade RW (1992). Limited MHC polymorphism in the southern elephant seal: implications for MHC evolution and marine mammal population biology. Proc $R$ Soc Lond Ser $B$ 249: $163-171$.

Slade RW, Moritz C, Heideman A, Hale PT (1993). Rapid assessment of single-copy nuclear DNA variation in diverse species. Mol Ecol 2: 359-373.

Taberlet P, Fumagalli L, Wust-Saucy A-G, Cosson J-F (1998). Comparative phylogeography and postglacial colonisation routes in Europe. Mol Ecol 7: 453-464.

Takahata N, Nei M (1990). Allelic genealogy under overdominant and frequency-dependent selection and polymorphism of major histocompatibility complex loci. Genetics 124: 967-978.

Trowsdale J (1995). 'Both man and bird and beast': comparative organization of MHC genes. Immunogenetics 41: 1-17.

Wu SK, Saunders TL, Bach FH (1986). Polymorphism of human IA antigens generated by reciprocal intergenic exchange between 2 DR-beta loci. Nature 324: 676-679. 